The 18th Annual IEEE International Symposium on Personal, Indoor and Mobile Radio Communications (PIMRC'07)

\title{
EFFECT OF METAL DOOR ON INDOOR RADIO CHANNEL
}

\author{
Jinwon Choi, Noh-Gyoung Kang, Jong-Min Ra, Jun-Sung Kang, and Seong-Cheol Kim \\ School of Electrical Engineering and Computer Science \& INMC, Seoul National University \\ Seoul, Korea
}

\begin{abstract}
This paper reports the variation of indoor radio channel caused by metal door. The simulation results using the Finite Difference Time Domain (FDTD) method and measurement results using the vector network analyzer in frequency domain are used for the characterization of received signal strength variation by metal door. Target frequency bands are three sensor band, 802.11b ISM band, and 802.11a UNII band. From the simulation and measurement results, the effect of door angle to the received signal strength in three frequency bands and effect of radio frequency to variation are investigated. And, FDTD simulation parameters for different environments are suggested.
\end{abstract}

\section{INTRODUCTION}

Deployment of wireless communication system is continuously growing in our homes and offices in last decade. To operate the efficient wireless communication systems, the accurate channel model is required at first. Literature has reported about the indoor channel model assuming static state as the fundamental resource for systems engineering $[1,2,3]$. However, though the access points and wireless communication devices stay stably, the wireless channel state can be changed as the physical environments near the receiver change. Among the many factors affecting to indoor wireless channel, the effect of door can be modelled using conventional simulation method and measurement. Though a few works have dealt with the related research about effect of door on the indoor radio channel [4, 5], proper channel variation model caused by metal door has not been investigated yet.

In this paper, we characterized the effect of metal door on the indoor radio channel variation through the simulation and measurement. The metal door connected to corridor at office environment is selected as the simulation and measurement environments with 38 receiver positions. Three frequency bands - sensor band, 802.11b band and 802.11a band - are selected for considering existing communications. For the analysis, the FDTD method is performed for simulation and $\mathrm{S}_{21}$ parameter of each receiver position is obtained at first. This simulation result is used to predict the variation of wireless channel by metal door. And, for the empirical characterization, channel transfer functions are measured using vector network analyzer in frequency domain. 200 channel transfer functions (CTFs) are collected at each receiver position and each frequency band. In measurement scenario, the angles of opened door are set to five representative values $-0^{\circ}, 30^{\circ}, 45^{\circ}, 60^{\circ}$, and $105^{\circ}$. The measured received signal strength values of frequency bands are modelled to the door angle and distance from the transmitter. After analyzing the Received Signal Strength (RSS) variation based on measurement results, the difference between simulation and measurement results is analyzed. Analyzing the reason why difference occurs, factors for more accurate simulation are suggested for further work in different environments.

The paper is organized as follows. After introducing the modeling environment, simulation algorithm, and measurement campaign in Section II, Section III analyzes simulation and measurement results. After Section VI compares the results and conclusion is followed in Section V.

\section{MODELING SPECIFICATIONS}

\section{A. Modeling Environment}

To characterize the effect of metal door on wireless channel exactly, we selected the modeling environment. In this environment, effect of other factors to indoor radio channel has to be negligible, except for that of metal door. So, office having metal door connected to the aisle of ferro-concrete building is selected for modeling. The ground plan of modeling environment is drawn in Figure 1 with transmitter and receiver positions. In this environment, an aisle is surrounded by the metal walls. So, the signal radiated from the transmitter over the door could propagate to the receiver via the door-path only. There are 38 receiver positions in aisle and the reference distance is set to $2 \mathrm{~m}$ because the $1 \mathrm{~m}$-sidth metal door opens toward transmitter. For the easy configuration of receiver points, the $\mathrm{V}$-line and $\mathrm{H}$-line is defined. V-line is composed of receiver positions with same distance from reference position (V-line 2: 2A, 2B, 2C, 2D, and $2 \mathrm{E}$ ) and $\mathrm{H}$-line possesses the receiver positions with same location (H-line A: 1A, 2A, , , and 8A).

\section{B. Modeling Environment}

Though ray tracing techniques can provide the accurate propagation model easily, it is not suitable to predict the diffraction of transmitted field by the metal door precisely. However, in numerical analysis from FDTD method, it is possible to investigate complex propagation behaviour near the metal door properly. The FDTD method is formulated by the differential form of Maxwell's two curl equations over a finite volume and approximating the derivatives with centred difference approximations [6,7]. For simplicity, the media are assumed uniform, isotropic, and homogeneous. With these assumptions, Maxwell's curl equation can be written as (1). In (1), $\vec{E}$ means electric field intensity, $\vec{H}$ is magnetic field intensity, $\mu$ is permeability and $\varepsilon$ denotes the permittivity. 


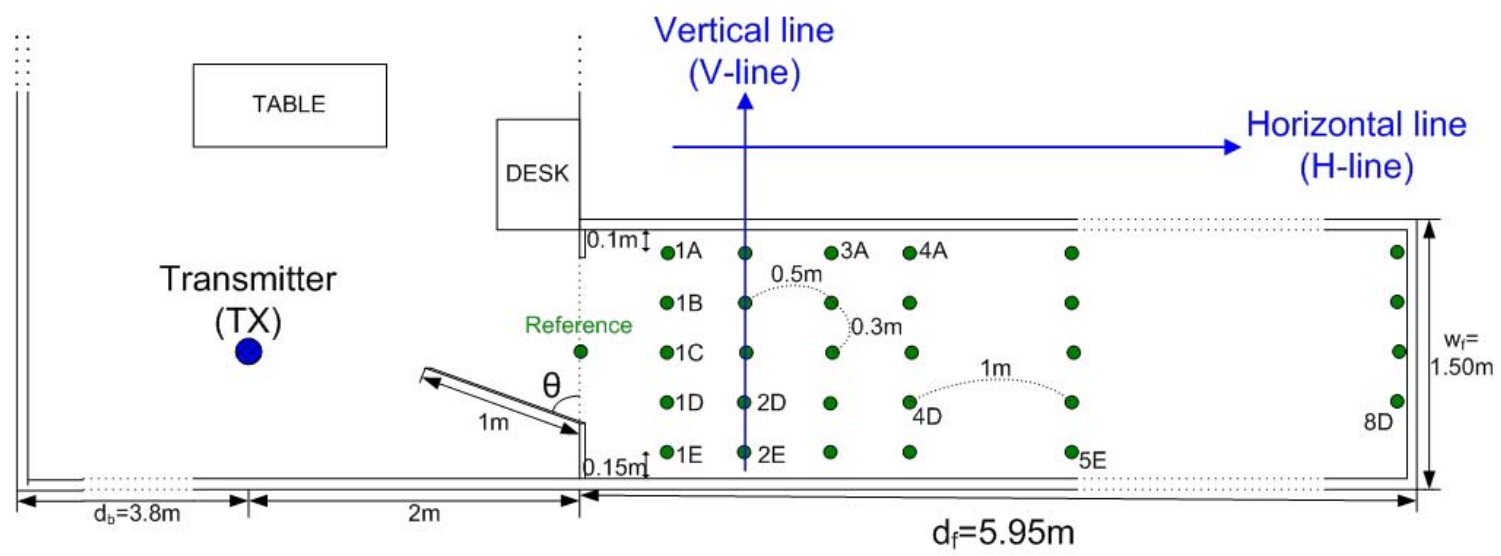

Figure 1: Top-view of transmitter and receiver positions with ground plan of measurement environment

$$
\begin{aligned}
& \mu \frac{\partial \vec{H}}{\partial t}=-\nabla \times \vec{E} \\
& \varepsilon \frac{\partial \vec{E}}{\partial t}=\nabla \times \vec{H}
\end{aligned}
$$

From this simple formula, propagating behaviour of transmitting signal is obtained. Fig. 2 illustrates the typical image of $E_{z}$ field propagation based on the FDTD simulation results using the sine-wave source. In simulation, cell size is set to $0.01 \mathrm{~m} \times 0.01 \mathrm{~m}$.
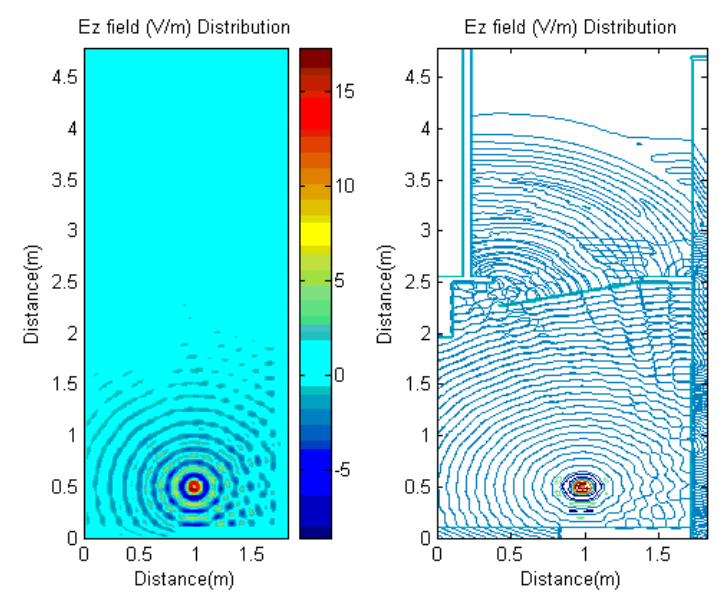

Figure 2: Picture of propagating $\mathrm{E}_{\mathrm{z}}$ field obtained using FDTD in measurement environment

\section{Modeling Environment}

For the statistical modeling of indoor radio channel, the channel measurement system based on frequency sweep method is constructed [8]. In this system, the vector network analyzer is used for channel characterization and power amplifier used to adjust the proper transmitting power. The target frequency ranges are for sensor band $(850 \sim 950 \mathrm{MHz})$,
IEEE $802.11 \mathrm{~b}$ ISM band $(2.4 \sim 2.5 \mathrm{GHz})$ and IEEE $802.11 \mathrm{a}$ UNII band $(5.15 \sim 5.35 \mathrm{GHz})$. In this paper, these frequency bands are denoted as S-Band, B-Band, and A-Band respectively. The resolution of measurement system is $1 \mathrm{MHz}$ in all frequency bands and the antennas are omni-directional ones with $3 \mathrm{~dB}$ gain on the tripod with height $1.5 \mathrm{~m}$. During measurements, environments near the receivers are kept stationary and people are not allowed to maintain the quasistatistic channel assumption. 200 channel transfer functions are collected in each receiver position and each frequency band. Time duration between consecutive CTFs is 1 second. Typical measured CTF is drawn in Fig. 3. In measurements, the doors are open with the specific angles, $\theta\left(0^{\circ}, 30^{\circ}, 45^{\circ}\right.$, $60^{\circ}$, and $\left.105^{\circ}\right)$. After measurements, all measured data are calibrated with the results at anechoic chamber.

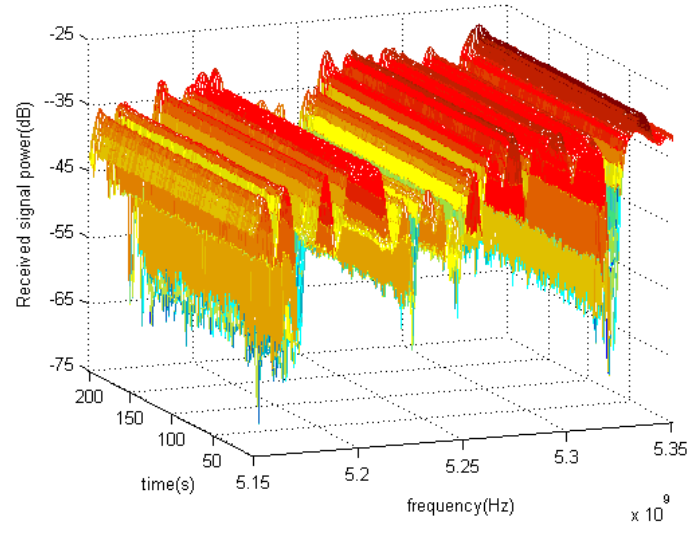

Figure 3: Typical measured channel transfer function of Aband during $200 \mathrm{~s}$ 


\section{RESULTS AND ANALYSIS}

\section{A. Simulation results}

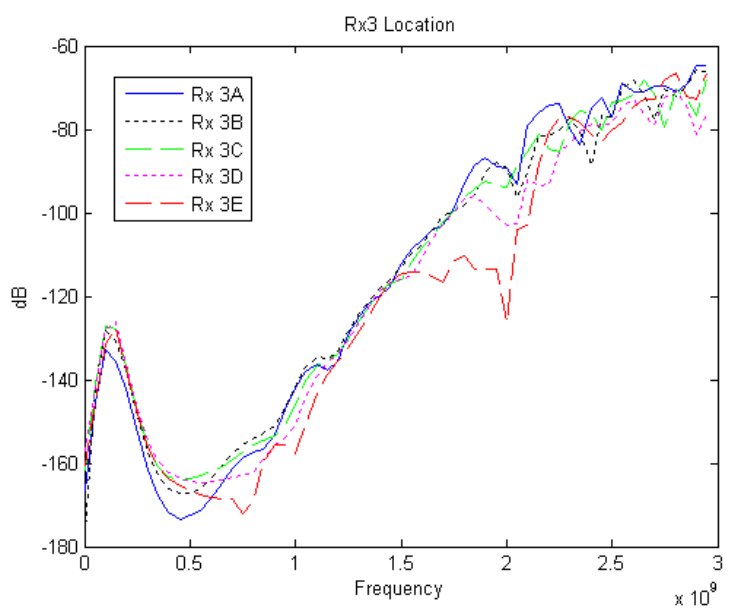

Figure 4: Pathloss variation of V-line 3 to frequency at door angle 30 degrees

Fig. 4 shows the FDTD results at receiver positions of $\mathrm{V}$ line 3 when the angle of door is set to 30 degrees. In this figure, the pathloss from transmitter is drawn in $\mathrm{dB}$ scale to the radio frequency. From this pathloss value to frequency, the RSSs correspond to three frequency bands are extracted and averaged. Fig. 5 indicates the typical RSS degradation behaviour predicted by FDTD results. This behaviour is that of B-Band and represented with door angle. In B-Band, the amount of RSS variation between widely opened door and closed door is predicted as $24 \mathrm{~dB}$. In the same say, the average values of RSS degradation are obtained as about $20 \mathrm{~dB}$ in SBand and $26 \mathrm{~dB}$ in case of A-Band. In the RSS analysis to door angle, it is predicted that the RSS decreases steeply between $40^{\circ}$ and $60^{\circ}$ and does not vary much below $40^{\circ}$.

And it is also predicted that the RSS decreases linearly with transmitter-receiver separation. The RSS of same V-Lines of Fig. 5 mean the RSS at same distance. The RSS difference is almost constant between different V-Lines. This is because, in the FDTD simulation, the medium between transmitter and receiver is homogenous and the signal penetration through the gap between door and wall is ignored.

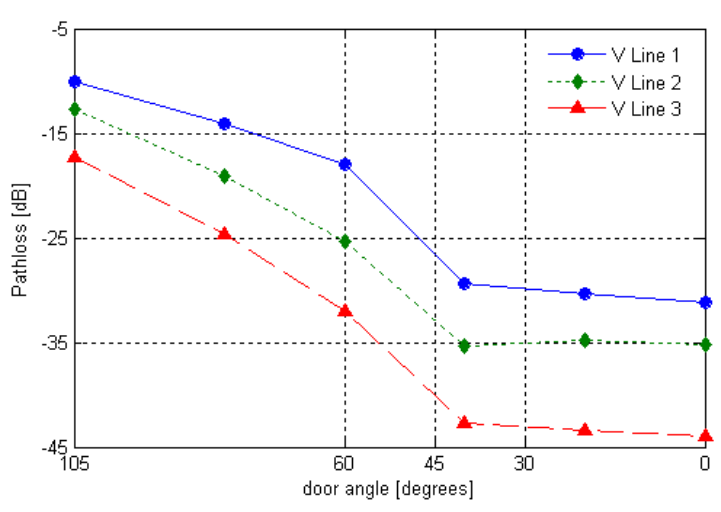

Figure 5: Typical simulation results about RSS degradation with decrease of metal door angle in B-Band

\section{B. Measurement results}

\section{1) Pathloss at reference distance}

From measured results, the variation of pathloss at reference distance $(=2 \mathrm{~m}), \operatorname{PL}\left(\mathrm{d}_{0}\right)$, to door angle is extracted at first. These values indicate the variation of RSS at the receiver point right behind of the metal door. Numerical results about variation of $\operatorname{PL}\left(\mathrm{d}_{0}\right)$ of three frequency bands are reported in Table 1 . In all frequency bands, $\operatorname{PL}\left(\mathrm{d}_{0}\right)$ decreases as metal door is closed. The amount of $\operatorname{PL}\left(\mathrm{d}_{0}\right)$ degradation to door angle is about $17 \mathrm{~dB}$ for S-Band, $29 \mathrm{~dB}$ for B-Band, and $26 \mathrm{~dB}$ for A-Band. This difference implies that the amount of diffraction caused by metal door is different when the operating wireless system is different.

And, the $\operatorname{PL}\left(\mathrm{d}_{0}\right)$ gap between different frequency bands in same door angle changes to the door angle. When the door is widely open, the difference between $\operatorname{PL}\left(\mathrm{d}_{0}\right)$ of S-Band and $\operatorname{PL}\left(\mathrm{d}_{0}\right)$ of A-Band is $14.35 \mathrm{~dB}$ then, as door gets closed, the difference grows to $23.39 \mathrm{~dB}$. This is because the RSS degradation by the metal door at higher frequency band gets bigger as angle of opened door, $\theta$, decreases.

Table 1: PL $\left(\mathrm{d}_{0}\right)$ of each frequency band in selected door angle

\begin{tabular}{|c||c|c|c|c|c||c|}
\hline$[\mathrm{dB}]$ & $\begin{array}{c}105^{\circ} \\
\text { (open) }\end{array}$ & $60^{\circ}$ & $45^{\circ}$ & $30^{\circ}$ & $\begin{array}{c}0^{\circ} \\
\text { (close) }\end{array}$ & $\Delta$ \\
\hline \hline S-Band & -53.55 & -59.18 & -59.64 & -65.23 & -70.23 & 16.68 \\
\hline B-Band & -56.61 & -65.87 & -69.83 & -77.98 & -85.60 & 28.99 \\
\hline A-Band & -67.80 & -75.24 & -82.13 & -87.12 & -93.62 & 25.82 \\
\hline \hline$\Delta$ & 14.35 & 16.06 & 22.49 & 21.89 & 23.39 & \\
\hline
\end{tabular}


2) Variation of RSS to the angle of opened door

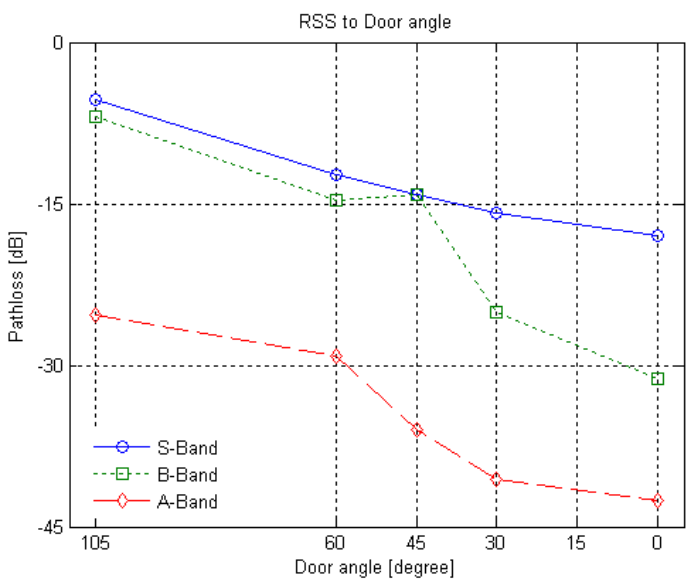

Figure 6: Measured results about RSS averaged over all receiver points as metal door is closed in three frequency bands

Fig. 6 shows the measurement results about RSS degradation to the change of $\theta$, angle of metal door. Values of Fig. 6 are averaged over all receiver positions. In, S-Band, RSS decreases linearly as door is closed. However, RSS of Bband drops steeply when door angle gets below 45 degrees and RSS of A-Band does when angle is below 60 degrees. The total difference between RSS of opened door and that of closed door is $14 \mathrm{~dB}$ of S-Band, $24 \mathrm{~dB}$ of B-Band, and $18 \mathrm{~dB}$ of A-Band respectively. If the effect of transmitter receiver separation is considered, the effect of metal door angle decrease as the receivers get further from the transmitter as reported in Table 2. At the right behind of the metal door, the variation is $20.6 \mathrm{~dB}$ and it decreases to $14.7 \mathrm{~dB}$ in A-Band. Though the results of A-Band are reported only in Table 2, the results of other frequency bands have the same tendency.

\section{3) Effect of $T x$-Rx separation on RSS}

Fig. 7 illustrates the average RSS of S-Band and A-Band to the distance from reference point to receiver point. All values are normalized to $\operatorname{PL}\left(\mathrm{d}_{0}\right){ }_{-} 105^{\circ}$ of Table 1 and value at specific distance means the RSS average over the V-line at same distance. Like previous analysis, in all frequency bands, the RSS when the door is widely open is the biggest and it decreases as metal door is closed. In S-Band, when the door is closed over 45 degrees, the RSS does not vary much. However, in case of A-Band, the RSS when angle of opened door is 45 degrees is about $10 \mathrm{~dB}$ bigger than RSSs when angle is 30 degrees or 0 degree.

To analyze the effect of transmitter-receiver separation on the RSS degradation, numerical measurement results are summarized in Table 2. The values of Table 2 are averaged over two neighboring V-Lines. When the door is widely opened, the difference of RSS at the nearest positions and that of the furthest positions is $4.8 \mathrm{~dB}$ while $1.2 \mathrm{~dB}$ when the door is closed. In case of the open angle of metal door is large, the effect of separation is large as well.

Table 2: RSS values averaged over V-Line $1 \& 2,4 \& 5$, and $7 \& 8$ with their gap in A-Band

\begin{tabular}{|c||c|c|c||c|}
\hline \multirow{2}{*}{$\mathrm{E}_{\text {RSS }}$} & \multicolumn{3}{|c||}{ V-Line } & \multirow{2}{*}{$\Delta$} \\
\cline { 2 - 4 } & 1,2 & 4,5 & 7,8 & \\
\hline \hline $105^{\circ}$ & -22.54 & -25.74 & -27.30 & 4.76 \\
\hline $45^{\circ}$ & -34.75 & -33.48 & -37.46 & 2.71 \\
\hline $0^{\circ}$ & -43.15 & -43.02 & -41.96 & 1.19 \\
\hline \hline$\Delta$ & 20.61 & 17.28 & 14.66 & \\
\hline
\end{tabular}

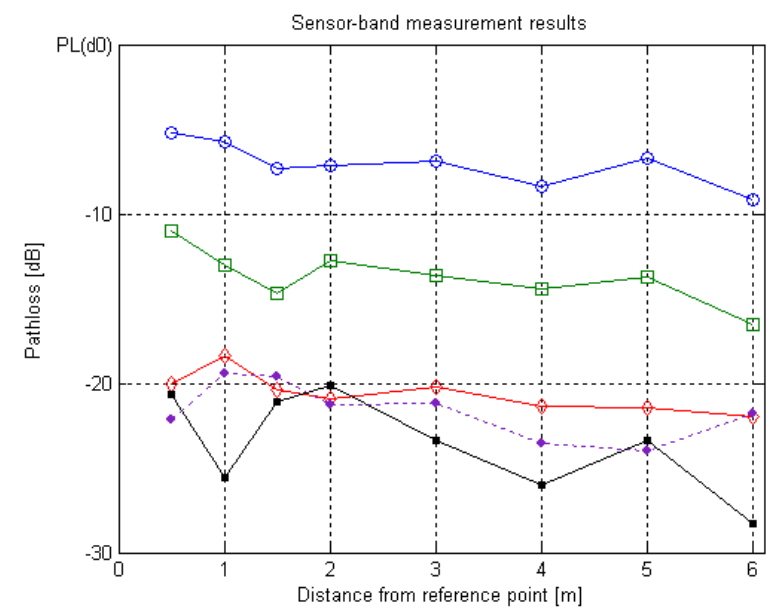

a) RSS of S-Band to the distance

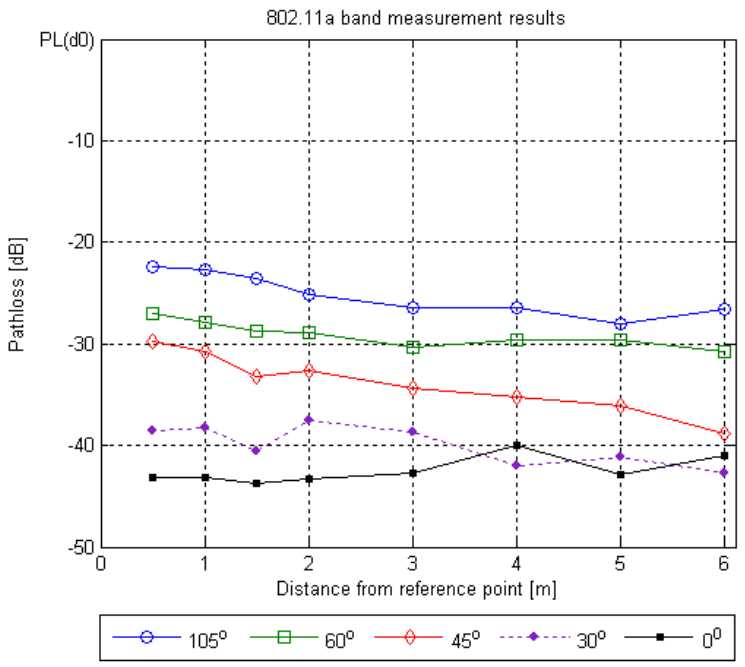

b) RSS of A-Band to the distance

Figure 7: Pathloss averaged over V-Line at A-Band to the separation with specific door angle 


\section{COMPARISON BETWEEN SIMULATION AND MEASUREMENT}

Table 3: Comparison between simulation and measurement results about degradation of RSS by metal door

\begin{tabular}{|c|c|c|c|}
\hline$\Delta$ RSS [dB] & $\begin{array}{c}\text { Simulation } \\
\text { Results (1) }\end{array}$ & $\begin{array}{c}\text { Measurement } \\
\text { Results (2) }\end{array}$ & $(1)-(2)$ \\
\hline S-Band & 20 & 14 & 6 \\
\hline B-Band & 24 & 24 & 0 \\
\hline A-Band & 26 & 28 & -2 \\
\hline
\end{tabular}

For the comparison, in Table 3, we list up the simulation and measurement results about degradation of RSS caused by open angle metal door. In other words, this degradation means the difference between RSS with opened door and RSS with closed door. For B-Band, two results are almost same, while difference is $6 \mathrm{~dB}$ of S-Band, $2 \mathrm{~dB}$ of A-Band. In lower band, the attenuation of transmitted signal is over-estimated because diffraction and penetration via door is calculated imperfectly. And, in higher frequency band, the attenuation of transmitted signal is under-estimated because of simulation assumption about wall material. The received signal is composed of multi-path signals which are multiply reflected to the surrounding walls. In simulation, these walls are assumed to be perfect conductor. However, the real walls are organized with metal boards. So, additional loss arises through the gap between metal boards,

In Fig. 8, comparison between measurement results and FDTD results along the V-line is illustrated. For the fair comparison, the received power signal levels of results are normalized. In both results, the received signal is the biggest in receiver A case and the lowest in E case. However, in measurement results, the received signal power decreases constantly as receiver moves from the A position to E position while the power fluctuates in middle positions of FDTD results. This means that the RSS of position behind door is smaller than those of other positions.

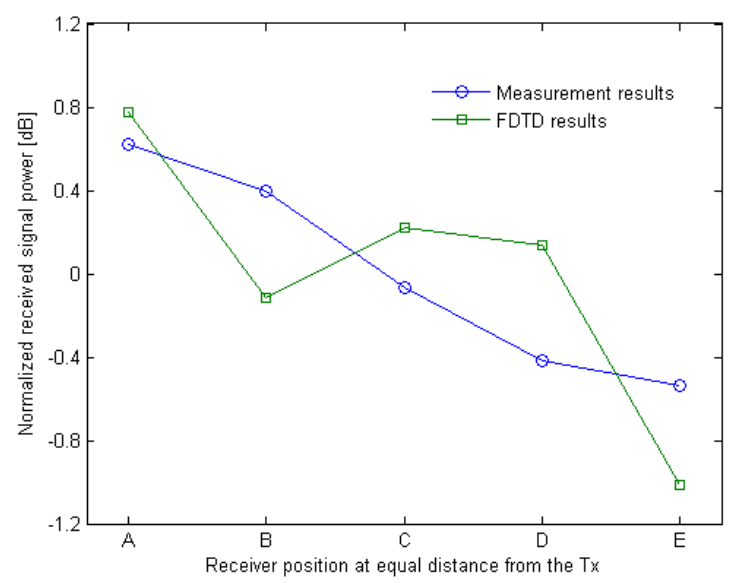

Figure 8: Comparison of normalized received signal power between positions along the $\mathrm{V}$-line

\section{CONCLUSION}

To characterize the effect of metal door on the indoor radio channel, numerical simulation and measurements are performed. Target frequency bands are $900 \mathrm{MHz}, 2.4 \mathrm{GHz}$, and $5.2 \mathrm{GHz}$ bands. Using FDTD simulation and measurement results, degradation of RSS caused by metal door is obtained as under $20 \mathrm{~dB}$ in $900 \mathrm{MHz}$ band and over $25 \mathrm{~dB}$ in other frequency bands. And as angle of opened door decrease below 60 degrees, the degradation is accelerated. Near the door, the degradation is bigger and as receiver goes away from the door, effect of door diminishes. In $2 \mathrm{GHz}$ band, the simulation and measurement results are perfectly matched while differences are observed in other bands. It seems that the accurate environment modeling is needed for more efficient modeling. For the future work, wideband channel characteristics like delay parameters will be discussed.

\section{ACKNOWLEDGMENT}

This work is partly supported by ETRI (Electronics and Telecommunications Research Institute) and Seoul R\&BD program (10544)

\section{REFERENCES}

[1] Borrelli, A., Monti, C., Vari, M.,and Mazzenga, F., "Channel models for IEEE 802.11 b indoor system design," in proc. of IEEE International conference on communications 2004, vol. 6, June, 2004, pp. 3701-3705.

[2] S.C.Kim, H. L. Bertoni, M. Stern, "Pulse propagation characteristics at $2.4 \mathrm{GHz}$ inside buildings", IEEE Journal on Vehicular Technology, vol 45, Issue 3, pp. 579 - 592, Aug 1996.

[3] Medbo. J, Berg. J.E, Harrysson, F., "Temporal radio channel variations with stationary terminal," IEEE Vehicular technology conference 2004, vol. 1, pp. 91-95, Sept. 2004.

[4] S. Collnge, G.Zaharia and G. El Zein, "Influence of the furniture on 60 $\mathrm{GHz}$ radio propagation in a residential environment," International Symposium on signals, circuits and systems 2003, vol. 20, Issue 6, pp.413-416, Jul. 2003.

[5] Sata, R., and Shirai, H., "Simplified analysis for indoor propagation of a WLAN channel," in proc. of IEEE Topical conference on wireless communication technology, Oct., 2003, pp. 248-249.

[6] Kane Yee, "Numerical solution of initial boundary value problems involving maxwell's equations in isotropic media," IEEE Trans. Antennas Propagation, vol.14, Issue 3, pp. 302-307, May 1966.

[7] Nam Koo Kang, Seong-Cheol Kim, Sangwook Nam, "Reflection coefficient calculation of a feeder structure in a RLSA," IEEE Antennas and Propagation Society International Symposium, pp.538-541, Jun. 2002.

[8] Jinwon Choi, Noh-Gyoung Kang, Yu-Suk Sung and Seong-Cheol Kim, "Empirical Ultra Wide Band pathloss model in office environments," IEEE Vehicular Technology Conference 2006, vol 4, pp. 156- 1960, May, 2006. 